\title{
MODELLING OF CRACK INITIATION IN ADHESIVELY BONDED JOINTS
}

\author{
H. Al Ali and M. Abdel Wahab \\ Ghent University, Laboratory Soete, Belgium
}

\begin{abstract}
In this paper, a review of some techniques proposed in the literature for modelling crack initiation in adhesively bonded joints is presented. The techniques reviewed are: a) the singular intensity factor, b) the inherent flaw size, c) Cohesive-zone model (CZM) and d) Continuum Damage Mechanics (CDM). The singular intensity factor characterizes the stress singularity at the corner point and can be used as a failure criterion to predict crack initiation. The inherent flaw method technique assumes that a small crack having a fraction of millimetres is initiated at the singular point in order to develop a fracture mechanics criterion for crack initiation. The strain energy release rate for an un-cracked specimen is used to determine the size of the inherent flaw. The cohesive zone model (CZM) technique is based on defining parameters from fracture mechanics test specimens and using them to model failure of the joints. Continuum Damage Mechanics makes use of thermodynamics principles in order to derive a damage evolution law. In this damage evolution law the damage variable $(D)$ is expressed as a function of number of cycles, applied stress range and triaxiality function. Furthermore, the possibility of using the eXtended Finite Element Method (XFEM) to predict crack initiation is elaborated.
\end{abstract}

Keywords: Crack initiation, adhesive, bonded joints, Fracture Mechanics, Finite Element Method, Continuum Damage Mechanics

\section{INTRODUCTION}

Adhesive bonding is currently gaining popularity in many advanced industrial applications such as aerospace, automobiles, ship structures, electronics, packaging, medical equipments and human bodies (dentistry and orthopedic), and shoe industries, etc. The main advantages of adhesive bonding compared to other joining techniques such as welding, riveting, bolting, and brazing are: a) the ability to join dissimilar materials and damage-sensitive materials, b) better stress distribution, c) weight reduction, d) fabrication of complicated shapes, e) excellent thermal and electrical insulation properties and f) greater fatigue resistance. The design of the joint is critical to the structural integrity and safety of building components. To date, various adhesive joints appear in the literature such as butt, cleavage, single lap and scarf joints. While enjoying much success, the use of each joint dependent on its application and therefore, many conditions have to be met to ensure no occurrence of failure. If we want to speak about the integrity of these joints, we must know that during its manufacturing process in the industry there will be cracks or flaws. This defect will be inevitable, and the presence of these flaws will affect the performance of adhesive bonding in service, or even cause catastrophic failure to the joint.

From the literature, Groth and Brottare [1] studied singular intensity factor for but joint using finite element analysis and they found that the stresses at the interface region between adhesive and substrate at the free edge can be described by a singular intensity factor $Q$. They also discovered that the fracture criteria related to $Q$ will be applicable for both linear elastic material and nonlinear plastic material.

Anderson and De Vries [2] have introduced another technique based on fracture mechanics, known as the "inherent flaw method ". In this method a small crack (a fraction of millimetres) is initiated at the singular point and strain energy release rates are measured. The size of this crack has to be defined experimentally by testing several specimens with different crack lengths and interpolating $a_{0}$ using the load of the untracked specimen. But the drawback of this method is the need of large number of experiments to determine the inherent crack size and the reduction of accuracy in introducing this very small crack. Further criticism on this method is that it may be not appropriate to represent an untracked 
specimen by a cracked one. However, the argument of Anderson and Devries suggested that the inherent flaws exist naturally in all bonds because of the bubbles of air.

Liljedahl et al [3] has studied the resistance of adhesively bonded aluminium, composite and dissimilar adherends joints exposed to humid environments. A cohesive zone model (CZM) technique was used to model failure of the joints, where the governing parameters were defined from fracture mechanics test samples appeased in a range of humid environments. The reduction in residual strength of aluminium single lap joints (SLJ) inundated in de-ionised water was predicted. For joints submerged in tap water the deterioration was faster than predicted and there were signs of corrosion. It was found that some disagreement between the experimental and the predicted data of the aged composite SLJ took place. The predicted deterioration overestimated the residual strength of the double lap joints (DLJ). It was suggested that this might be because of a residual stress-enhanced degradation mechanism. Large residual stresses were induced in the dissimilar adherends joints caused by the mismatch of coefficients of expansion of the substrates.

In the past, the Finite Element Method (FEM) has been used for the simulation and strength prognostication of bonded joints. Cohesive-zone models (CZMs) were proved to be a powerful technique in modelling damage growth, surpassing some restriction of the FEM. In spite of this fact, they still have the constraint of damage growth only at a known growth path [3]. The eXtended Finite Element Method (XFEM) is a new amelioration of the FEM, developed to allow the growth of discontinuities in bulk solids along an arbitrary path, by enriching degrees of freedom with special displacement functions. With this technique, solution for the main restriction of CZMs could be found [4].

\section{SINGULAR INTENSITY FACTOR}

Groth and Brottare [1] studied singular intensity factor for butt joints using finite element analysis. Close to the singularity at the free edge of the joint, the stresses are characterized by:

$$
\sigma_{y}=Q_{y}\left(\frac{r}{h}\right)^{-\lambda}, \lambda>0
$$

Where $\lambda$ the strength of singularity and QY is is the singular intensity factor. Equation (1) is applicable to elastic and elasto-plastic materials. The size of plastic zone is determined by:

$$
\frac{\mathrm{r}_{\mathrm{s}}}{\mathrm{h}}=\left(\mathrm{Q}_{\mathrm{y}} / \sigma_{\mathrm{s}}\right)^{1 / \lambda}
$$

Gradin and Groth $[5,6]$, in their work, which concerns with the finite element analysis, they considered the wedge angles shown in Figure 1, where $\alpha_{1}$ and $\alpha_{2}$ equals to $\pi / 2$. By using the displacement near the singularity, the singular intensity factor $Q_{Y}$ can be obtained from FE results. The expression of $Q_{Y}$ can be re-arranged from Equation (1) as:

$$
Q_{y}=\frac{(1-\lambda)}{h\left(\frac{y}{h}\right)^{(1-\lambda)}\left(1-v^{2}\right)}\left(u_{y}-u_{0}\right)
$$

Equation (3) is acceptable for linear elastic material. $u_{0}$ is the displacement in the $y$ direction at the singular point $(x=0)$ and $u_{y}$ is the displacement in $y$-direction for ant coordinate $x$. Plots of $Q_{y}$ versus $\frac{y}{h}$ (the non-dimensional coordinate) for different materials are shown in Figure 2. From Figure 2, it can be seen that for all non-linear materials when $\frac{y}{h} \rightarrow 0, Q_{y}$ goes to infinity. For the linear elastic case, when $\frac{y}{h} \rightarrow$ 0 , then $Q_{y}$ equals to $13.6 \mathrm{MPa}$. By considering $Q_{y}$ at some distance from the singularity point, it is possible to evaluate $Q_{y}$ for nonlinear materials. 


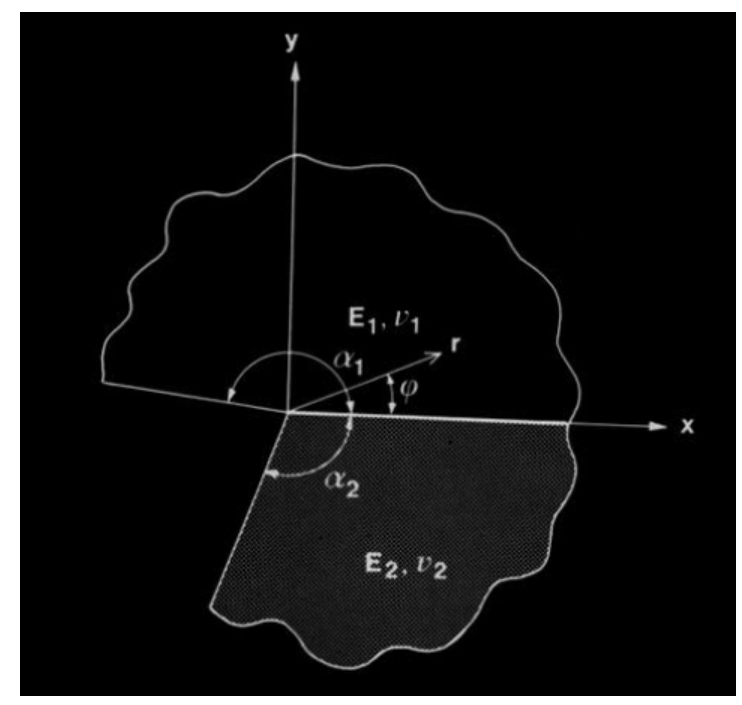

Figure 1. Geometry of bi-material wedge $[5,6]$

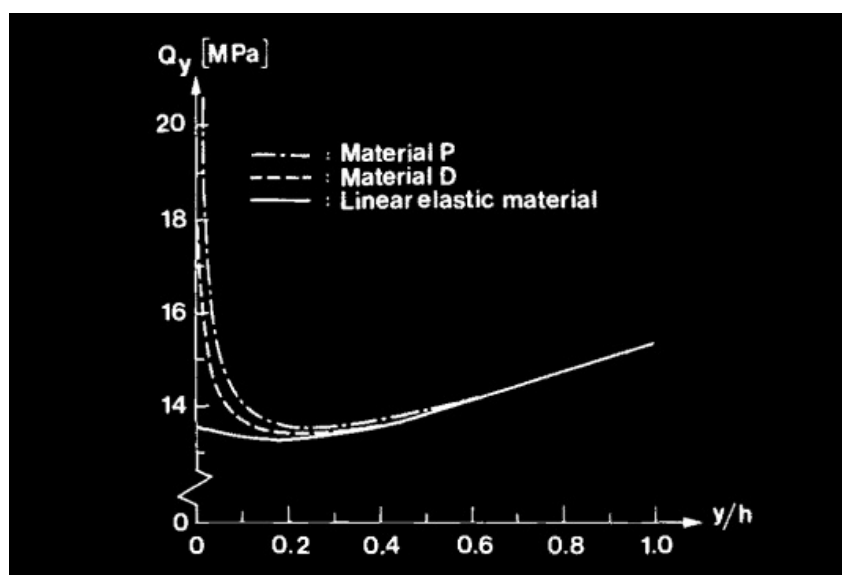

Figure 2. Singular intensity factor for different materials [1]

It is important to know the size of the plastic zone in order to characterize failure in elasto-plastic materials. A first strict expression of the plastic zone size may be obtained by supposing a stress and stain field of the bi-material wedge shown in Figure 1. The first form of the stress filed is given by equation (1), while the plastic zone size $r_{s}$ is given by equation (2) for the case of elastic perfectly plastic material. In such a case the material starts to yield when $\sigma_{y}=\sigma_{s}$. This is shown in Figure 3 when the stresses above $\sigma_{s}$ are cut down in this case. From the nonlinear FE analysis, the plastic zone size is obtained. Figure 4 shows the definition of plastic zone sizes, where $r_{s}$ is given by equation $(2), r_{\max }$ is the maximum size of plastic zone with angle $\theta_{m}$ and $r_{y}, r_{x}$ are the size of plastic zone where $\mathrm{x}=0, \mathrm{y}=0$, respectively. The results showed that for all materials the estimated plastic zone $r_{s}$ for $s_{n o m}<0.8 \%$ was larger than the maximum plastic zone.

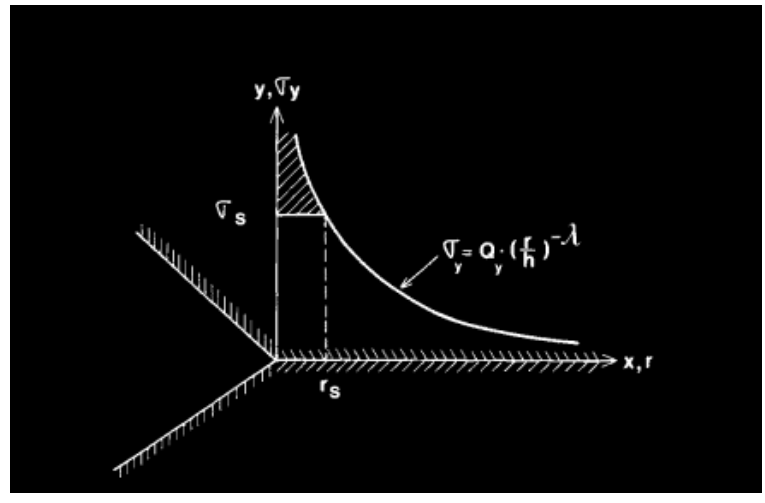

Figure 3. Normal stresses along the bi-material plane and $1^{\text {st }}$ approximation of plastic zone size [1]

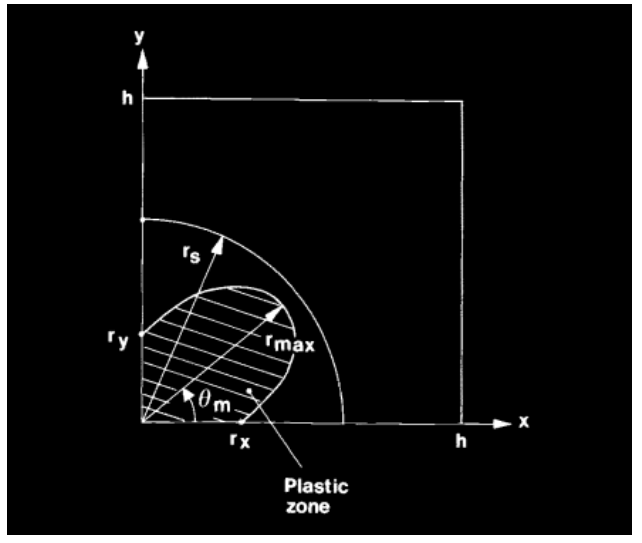

Figure 4. Definition of plastic zone sizes [1]

The angle, at which the maximum plastic zone size was located, ranged from 30 to $45^{\circ}$ and would converge to $45^{\circ}$ when the materials became near the linear elastic curve. The stresses at the free edge $\sigma_{y}$ may be reached by two ways for the linear elastic case; the first one from FE model and the second one from equation (1). It was found that, the maximum plastic zone size for valid small scale yielding equal to $0.03 \mathrm{~mm}$, in the condition of supposing that $r_{Q}$ was ten times larger than $r_{s}$. This was identical to a nominal strain level around $0.45 \%$. 
With the concept of $Q_{Y}$, a failure criterion can be developed. It is assumed that the fracture will start in the samples as soon as a critical value of the singular intensity factor is achieved, i.e.

$$
\begin{aligned}
& Q_{y}<Q_{\text {crit }}=\text { no fracture } \\
& Q_{y} \geq Q_{\text {crit }}=\text { fracture initiation }
\end{aligned}
$$

Where $Q_{\text {crit }}$ is the critical value of the singular intensity factor. A failure criterion given by equation (4) can be applied under small scale yielding condition.

\section{INHERENT FLAW METHOD}

Anderson and De Vries [2] have introduced another technique to predict crack initiation based on fracture mechanics, known as the "inherent flaw method ". In this method, a very small crack is initiated at the singular point and strain energy release rates are measured. The size of this crack has to be defined experimentally by testing several specimens with different crack length and interpolating $a_{0}$ using the load on the untracked specimen. One of the drawbacks of this method is the need of big number of experiments to determine the crack size. An inherent flaw size $\left(a_{0}\right)$ is defined as the amount of de-bond needed to product the suitable critical energy release rate at the measured peak load in samples with no initial de-bond. By using the inherent flaw size and the critical energy release rate, the strength in many bonded joints can be predicted. It was found that, adhesive thicknesses between $0.15 \mathrm{~mm}$ and $25 \mathrm{~mm}$ introduce failure loads varying from $1900 \mathrm{~N}$ for thin bonds to $180 \mathrm{~N}$ for thick bonds, respectively, as shown in Figure 5. For joints with adhesive layer thicker than $2.5 \mathrm{~mm}$, adhesive failure started at the bond edge while for joints with adhesive layer thinner than $2.5 \mathrm{~mm}$, it will be initiated near the centreline of the joints. The strain energy release rate was obtained using finite element method. In order to determine the inherent flaw size, the critical energy release rate was plotted against the crack length as shown in Figure 6 . Using the strain energy release rate the un-cracked specimen, an inherent flaw size was predicted. In most cases failures were cohesive into the adhesive layer near the bond edge. It was then assumed that failure initiated at the adhesive/adherend interface adjacent to the bond edge, then propagated into the adhesive and continued through the centre of the adhesive layer.

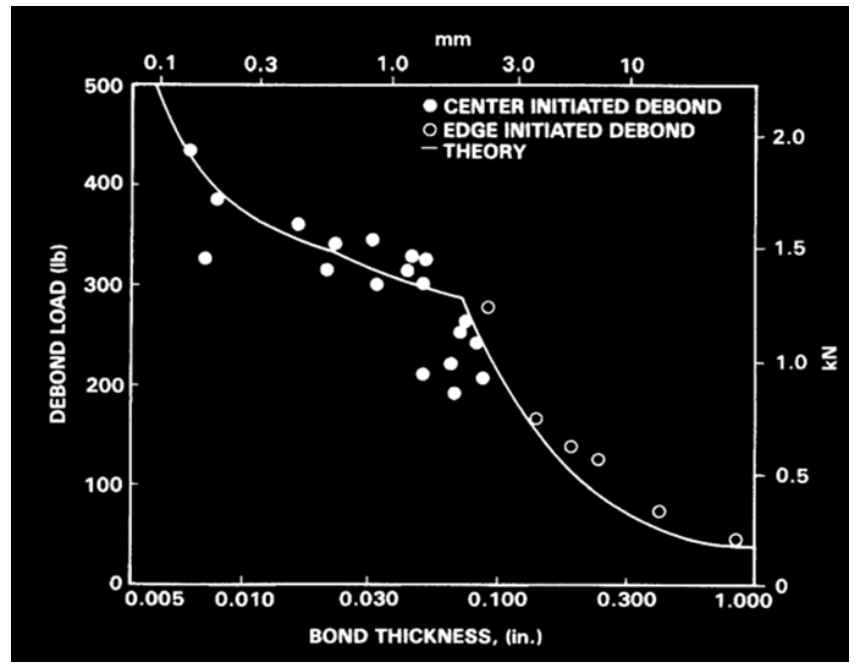

Figure 5. Effect of adhesive thickness on de-bond load [2]

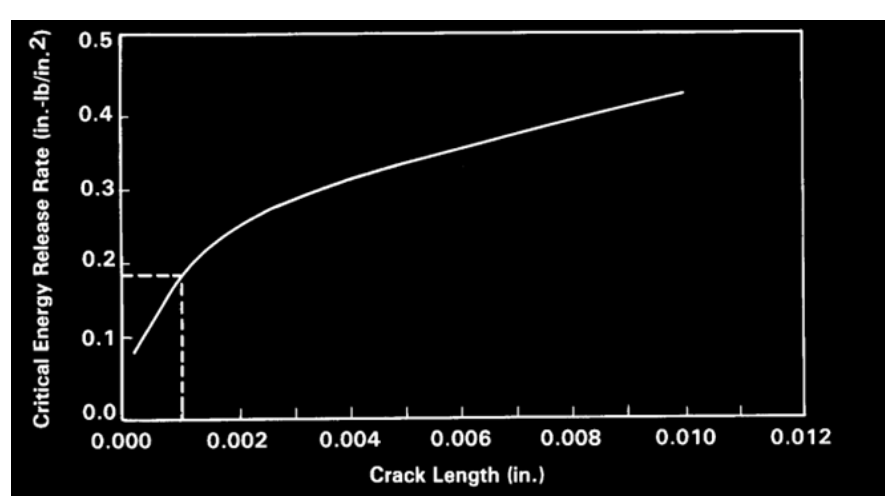

Figure 6. Critical strain energy release rate as a function of crack length [2] 
Furthermore, Anderson and De Vries [2] have tested non-tapered double cantilevered beams. In these experiments, a tensile force normal to the bond line of two parallel plates was applied and the crack was propagated incrementally by loading and unloading process. Then a load-displacement curve was obtained for different crack lengths. From these results the change in compliance of the specimen according to the change in crack length may be calculated. This allows calculation of $G_{c}$ as:

$$
G_{C}=\frac{P^{2}}{2 b} \frac{d c}{d a}
$$

Where $p$ is the crack propagation load, $b$ is the specimen width and $d c / d a$ is the change in compliance with crack length.

\section{COHESIVE ZONE MODEL}

Liljedahl et al [7] have used a cohesive zone model (CZM) technique to model failure of adhesive joints, where the governing parameters were defined from fracture mechanics test samples. A range of aging humid environments were considered. Different types of joints were tested, namely single lap metallic joints with Aluminium substrates (Figure 7(a), Al-Al SLJ), single lap composite joints with Carbon Fibre Reinforced Plastic substrates (Figure 7(b), CFRP-CFRP SLJ) and double lap metal to composite joints with one substrate Aluminium and one Carbon Fibre Reinforced Plastic (Figure 7(c), Al-CFRP DLJ). The failure of the joints was simulated using a CZM. A two-parameter CZM was used where division in mode I, II and III were accounted for. The CZM parameters are the fracture energy $\left(\Gamma_{0}\right)$ and the tripping traction $\left(\sigma_{u}\right)$ as indicated in Figure 8. The stiffness before unloading was set high to obviate any considerable compliance of the CZM element before the beginning of damage. For all numerical modelling work, the commercial FE package, ABAQUS (Hibitt, Karlsson \& Sorensen, Inc) was used. Non-linear springs (ABAQUS 6.5: 18.1.1) were used to model the traction-separation law at the interface. CZM is CPU intensive and it was therefore comfortable to model the joints in $2 \mathrm{D}$ if convenient. Large diffusion and stress analysis without CZM elements were thus done to use a range of 2D and 3D elements, with and without residual strains to decide whether 2D models provide good approximation or not. This was discussed further according to each joint formalization. Firstly, the CZM parameters were established for different moisture concentration using the MMF (Mixed Mode Flexure) and NCA (Notched Coated Adhesive) specimens. These parameters were then used to prognosticate the response of the SLJ and DLJ joints. The adhesive continuum was modelled using a linear Drucker-Prager model with a friction angle of 32.51 [7] and moisture-dependent hardening, predicted experimentally from the stress-strain test of bulk adhesive samples.

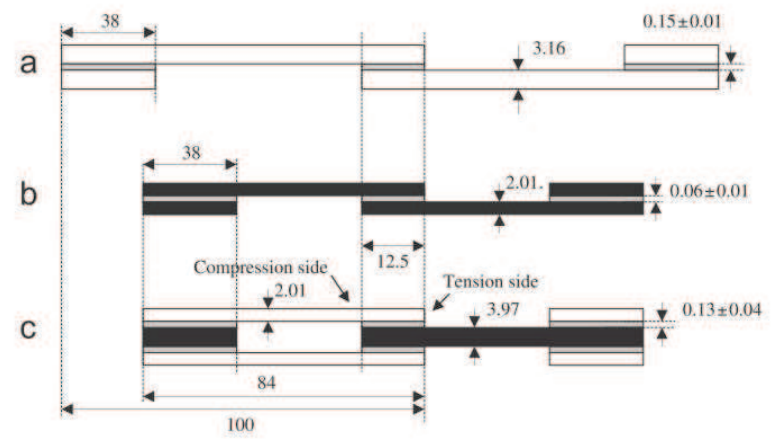

Figure 7. Dimensions of the lap joints, width $25 \mathrm{~mm}$, (a) Al-AI SLJ, (b) CFRP- CFRP SLJ and (c) AlCFRP -AI DLJ. (All dimensions in $\mathrm{mm}$ ) 「71

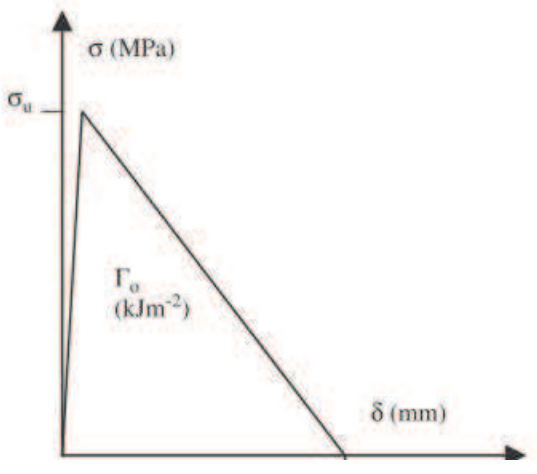

Figure 8. Cohesive zone model [7] 


\section{CONTINUUM DAMAGE MECHANICS}

Based on thermodynamics principles, a damage evolution law was derived by Abdel Wahab et. al [8]. The damage variable $(D)$ as a function of number of cycles, applied stress range and triaxiality function is given by:

$$
D=1-\left[1-A(\beta+m+1)\left(\Delta \sigma_{e q}\right)^{\beta+m} R_{V}^{\beta / 2} N\right]^{\frac{1}{\beta+m+1}}
$$

Where $D$ is the damage variable, $N$ is the number of cycles, $\Delta \sigma_{e q}$ is the range of von-Mises stress, $R_{V}$ the triaxiality function, $m$ the power constant in Ramberg-Osgood equation and $A$ and $\beta$ are damage parameters to be determined experimentally. For a strain-based controlled test, the damage variable $D$, can be determined as:

$$
D=1-\frac{\Delta \sigma}{\Delta \sigma^{*}}
$$

Where $\Delta \sigma_{e q}^{*}$ is von-Mises stress range for virgin material (undamaged state) or at stabilization of harding. The damage parameters $A$ and $\beta$ can be calculated for each applied load level by curve fitting Equation (6) to the experimental results, determined by Equation (7). In order to use the damage evolution law, the triaxiality function is required and, therefore, two types of stress should be determined from FEA; namely von Mises equivalent stress and Hydrostatic Stress. The triaxiality function is defined as $\sigma_{e q}\left(R_{v}=\left(\frac{\sigma^{*}}{\sigma_{e q}}\right)^{2}\right)$, and can also be written as $R_{v}=\frac{2}{3}(1+v)+3(1-2 v) T_{x}^{2}$, Where $v$ is Poisson's Ratio and $T_{x}$ is the triaxiality ratio $\left(\frac{\sigma_{H}}{\sigma_{e q}}\right)$. At failure, when the number of cycles to failure, $N_{f}$, is reached, $D$ becomes 1 (fully damaged state) so that fatigue lifetime can be predicted as [8]:

$$
N_{f}=\frac{\Delta \sigma_{e q}^{-\beta-m} R_{v}^{-\frac{\beta}{2}}}{A(\beta+m+1)}
$$

By curve fitting of Equation (8) to experimental results, the damage parameters $A$ and $\beta$ can be determined as function of applied stress $\Delta \sigma_{e q}$.

\section{EXTENDED FINITE ELEMENT}

In the past, the Finite Element Method (FEM) has been used for the simulation and strength prognostication of bonded joints. Cohesive-zone models (CZMs) were proved to be a powerful technique in modelling damage growth, surpassing some restriction of the FEM. In spite of this fact, they still have the constraint of damage growth only at a known growth paths. The eXtended Finite Element Method (XFEM) is a new amelioration of the FEM, developed to allow the growth of discontinuities in bulk solids along an arbitrary path by enriching degrees of freedom with special displacement functions. Using this technique, solution for the main restriction of CZMs might be found. Campilho et al. [4] have applied XFEM to predict the strength of adhesive joints and compared the results to CZM technique. In applying XFEM to fracture mechanics, enrichment functions for nodal displacements are used to simulate the separation of crack faces and the crack tip behaviour. The elements along the crack faces are enriched with a discontinuous function, while the elements at the crack tip are enriched with a near-tip asymptotic displacement function. For Linear Elastic Fracture Mechanics, the XFEM displacement field is approximated as: 


$$
U=\sum_{i \in \mathcal{N}} N_{i}(x) U_{i}+\sum_{i \in \mathscr{N}_{\text {cut }}} N_{i}(x) H(x) a_{i}+\sum_{i \in \mathscr{N}_{\text {front }}} \sum_{\alpha} N_{i}(x) B_{\alpha}(x) b_{i, \alpha}
$$

$N$ is the number of the standard finite element nodes, $N_{\text {cut }}$ the number of nodes which belong to elements completely cut by the crack faces and $N_{\text {front }}$ the number of nodes containing a crack front. $N_{i}$ are the standard finite element shape functions, $H$ is a function which value is 1 if $x$ is above the crack surface and -1 if $x$ is under the crack surface. For LEFM:

$$
\left[B_{\alpha}\right]=\left[\sqrt{r} \sin \frac{\theta}{2}, \sqrt{r} \cos \frac{\theta}{2}, \sqrt{r} \sin \frac{\theta}{2} \sin \theta, \sqrt{r} \cos \frac{\theta}{2} \sin \theta\right]
$$

For crack initiation, an equation similar to Equation (9) may be proposed by adding enriched functions that approximate stress singularity, plasticity, damage evolution (Equation (6)) and weak interface ahead of the singular point.

\section{REFERENCES}

[1] Groth, H. L., Brottare, I., journal of Testing Evaluation, 291, 1988.

[2] Anderson, G. P., DeVries, K. L., International Journal of Fracture 39, 191, 1989.

[3] Liljedahl, C.D.M. et al., International Journal of Adhesion \& Adhesives 27, 505-518, 2007.

[4] Campilho, R., Banea, M., Pinto, A., daSilva, L., deJesus, A., Strength prediction of single- and doublelap joints by standard and extended finite element modelling, International Journal of Adhesion \& Adhesives 31, 363-372, 2011.

[5] Gradin, P., A Fracture Criterion for Edge-Bonded Bi-Material Bodies, Journal of Composite Materials, 16(6), 448-456, 1982.

[6] Groth, H., A Method to Predict Fracture in an Adhesively Bonded Joint, International Journal of Adhesion and Adhesives, 5(1), 19-22, 1985.

[7] Liljedahl C.D., Crocombe A.D., Wahab M.A., Ashcroft I.A., International Journal of Fracture,141-147, 2006.

[8] Abdel Wahab, M.M., Ashcroft, I.A., Crocombe, A.D., Shaw, S.J., Prediction of fatigue thresholds in adhesively bonded joints using damage mechanics and fracture mechanics, Journal of Adhesion Science Technology, 15(7), 763-782, 2001. 